\title{
Recovering from Cystectomy: Patient Perspectives
}

\author{
Carmit K. McMullen ${ }^{\mathrm{a}, *}$, Marilyn L. Kwan ${ }^{\mathrm{b}}$, Janice C. Colwell ${ }^{\mathrm{c}}$, Julie R. Munneke ${ }^{\mathrm{b}}$, James V. Davis ${ }^{\mathrm{a}}$, \\ Alison Firemark ${ }^{\mathrm{a}}$, Neon Brooks ${ }^{\mathrm{a}}$, Marcia Grant ${ }^{\mathrm{d}}$, Scott M. Gilbert ${ }^{\mathrm{e}}$ and Andrea Altschuler ${ }^{\mathrm{b}}$ \\ ${ }^{a}$ Center for Health Research, Kaiser Permanente, Portland, OR, USA \\ ${ }^{\mathrm{b}}$ Division of Research, Kaiser Permanente, Oakland, CA, USA \\ ${ }^{\mathrm{c}}$ University of Chicago Medicine, Chicago, IL, USA \\ ${ }^{\mathrm{d}}$ City of Hope, Duarte, CA, USA \\ e Moffitt Cancer Center and Research Institute, Tampa, FL, USA
}

Received 19 October 2018

Accepted 10 January 2019

\begin{abstract}
.
Background: Bladder cancer patients who undergo cystectomy and urinary diversion face functional and quality-of-life challenges. Little is known about these patients' experiences during decision-making, surgery, and recovery, or how they vary by treatment setting.

Objective: To learn about patients' experiences with treatment choice, surgical care, and recovery across health settings. Understanding patient experiences is essential to closing care gaps and developing patient-reported measures.

Methods: We conducted focus groups with cystectomy patients and family caregivers at a large comprehensive health care system ( $\mathrm{N}=32$ patients) and an NCI-designated comprehensive cancer center ( $\mathrm{N}=25$ patients and 5 caregivers). Using standard qualitative methods, we identified themes that are not well-represented in existing research.

Results: Across both systems, patients described variable experiences in decision-making about their cystectomy and urinary diversion. Some felt overwhelmed by information; others felt poorly informed. Many found self-care equipment challenging; many felt they knew little about what to expect regarding chemotherapy, recovery, and transitioning home. At times, health care personnel could not help manage patients' ostomies or catheterization equipment. Our study also contributes a grounded theoretical framework for describing meaningful domains of patient experience with cystectomy and urinary diversion. We identified a common trajectory that includes decision-making, surgery and post-operative recovery, mastery of self-care, and reintegration.

Conclusions: Patients with radical cystectomy and urinary diversion report a wide variety of experiences not captured by quantitative measures. These findings demonstrate that many cystectomy patients could benefit from additional post-operative support. We offer a framework to measure patient-centered domains in future research.
\end{abstract}

Keywords: Patient-reported outcomes, bladder cancer, cystectomy, qualitative methods, focus groups

\footnotetext{
${ }^{*}$ Correspondence to: Carmit K. McMullen, Kaiser Permanente Center for Health Research, 3800N. Interstate Avenue, Portland, OR 97217, USA. Tel.: +1 503335 6381; Fax: +1 503335 6311; E-mail: Carmit.McMullen@kpchr.org.
}

\section{INTRODUCTION}

Bladder cancer patients who undergo cystectomy and urinary diversion face persistent functional impairments and poor health-related quality-oflife [1-11]. A recent stakeholder-driven research 
prioritization study involving bladder cancer patients and caregivers identified the need for more information about urinary diversion choice, cystectomy education, expectations about recovery, and impacts of cystectomy and urinary diversion [12]. Qualitative research using grounded theory approaches can provide these needed insights into common experiences, trajectories and transitions in illness experience [13] for bladder cancer patients undergoing cystectomy.

The few existing qualitative studies of patients who have undergone cystectomy and urinary diversion explored their decision-making processes and unmet needs over the course of treatment, but only in the context of academic medical centers [14-16]. Understanding the full range of patient experiences and concerns, and how they might vary across treatment contexts, is an essential precursor to identifying ways that health systems can effectively support patients throughout their treatment journey and developing objective measures that compare patient outcomes across choice of urinary diversion.

In this study, we conducted focus groups and indepth interviews with bladder cancer patients treated with radical cystectomy and urinary diversions in both (1) a large integrated health care delivery system, and (2) a comprehensive cancer center. We sought to identify patients' needs and challenges from pretreatment to approximately two years after surgery, and to identify how these needs were addressed across two different delivery systems.

\section{MATERIALS AND METHODS}

\section{Theoretical approach and design rationale}

We conducted focus groups in two health center contexts in order to develop a framework for research and practice improvement derived from patients' and caregivers' experiences with cystectomy and urinary diversion. Our study design, data collection, and analysis were based on modified grounded theory $[17,18]$.

In the first phase of data collection, we conducted focus groups with patients who had undergone urinary diversions at Kaiser Permanente Northern California (KPNC). We used a purposeful sampling strategy $[19,20]$ which we have employed previously in the colorectal cancer context [21] and assigned patients into focus groups by diversion type and gender, since both these variables were likely sources of variability in experience.
In the second phase, we conducted focus groups at Moffit Cancer Center. To more fully capture the family/caregiver experience, we included both patients and caregivers in these focus groups. We also decided to shift our attention to areas of common experience across diversion types. Therefore, those focus groups included patients and caregivers of mixed gender and urinary diversion type. The main source of variability of interest in this analysis, the health system context, was accounted for by collecting and analyzing data using similar methods from patients in two health systems. Exploring differences in experience by urinary diversion type and gender was not the focus of this analysis, but we did obtain data from each of these perspectives to ensure that our framework included experiences of male and female patients with different diversion types.

\section{Integrated health care system: Kaiser Permanente Northern California (KPNC)}

$\mathrm{KPNC}$ is a comprehensive medical system serving over 4.2 million members throughout Northern California. Patients were eligible for study participation if they were at least 21 years old at the time of surgery, resided in the greater San Francisco Bay Area, and received a cystectomy with urinary diversion (ileal conduit, neobladder, or continent pouch) for treatment of bladder cancer at KPNC between January 2007 and August 2012. Patients were not required to be Kaiser Permanente health plan members at the time of the study. We identified 303 eligible patients using electronic research databases derived from health plan data. We confirmed each patient's type of urinary diversion through chart review.

We emailed each eligible patient's urologist to ask permission to contact their patient(s). If the physician did not deny permission within two weeks, we mailed the patient an invitation letter and a postcard that interested patients could return. A study coordinator called patients who returned the postcard to schedule focus groups. We sent a reminder letter with the date, time, and location of the focus group to all patients who agreed to participate. Recruitment efforts continued until at least ten patients signed up for each focus group. We aimed to recruit at least two members of minority racial/ethnic groups in each focus group. Several recruited participants did not come to the focus groups, and we were not able to recruit ten women with neobladder or continent pouch diversions. Ultimately, we collected data from 
32 enrolled patients: nine men with an ileal conduit, nine men with a neobladder, nine women with an ileal conduit, and five women with a neobladder or continent pouch. Focus groups were scheduled for all groups except women with neobladder diversions: Because of the small number of patients in this group, we interviewed each participant individually by telephone. All data collection occurred in the spring of 2013.

\section{Comprehensive cancer center: Moffitt cancer center}

Moffitt Cancer Center is an NCI-designated comprehensive cancer center based in Tampa, Florida that serves the Southeastern United States. Moffitt's primary service area covers over 5 million individuals. Patients from Moffitt were eligible for recruitment if they were at least age 21 at the time of surgery and received a cystectomy and urinary diversion (ileal conduit, neobladder, or colon pouch) for treatment of bladder cancer between January 2011 and April 2016. We identified 484 eligible participants treated with cystectomy within this timeframe. A subgroup of patients who lived within 60 miles of the center were then prioritized for recruitment and contacted by a study coordinator who introduced the study and screened for eligibility. A total of 76 patients were reached, and 35 agreed to participate. They were scheduled to attend one of four focus groups based on what time was most convenient for them, and they were invited to bring a caregiver along to participate. Twenty-five patients and five caregivers arrived for the focus groups and all were consented and enrolled in the study during the day of the focus group. This resulted in the recruitment of 15 men with an ileal conduit, six men with a neobladder, three women with an ileal conduit, and one woman with a neobladder or continent pouch. Five caregivers participated in the focus groups, including one adult child and four spouses of participating patients.

The Institutional Review Boards at KPNC and KP Northwest reviewed and approved the study involving focus groups at KPNC. Moffitt Cancer Center approved of the study involving focus groups at Moffit Cancer Center. All procedures performed were in accordance with the ethical standards of the institutional and national research committee and with the 1964 Helsinki declaration and its later amendments or comparable ethical standards.

\section{Focus groups and interviews}

We used an interview guide for the focus groups and individual interviews that was developed based on previous literature as well as input from consulting urologists and ostomy nurses. Interviews began by eliciting general comments about diagnosis and treatment decision-making, and then asked about more specific experiences with surgery and recovery, as well as challenges and adaptations (see Fig. 1 for specific discussion prompts). At Moffit Cancer Center, we also asked questions about whether an online informational intervention would be useful for supporting patients and families before and after cystectomy (subsequent discussions about the informational intervention were not the focus of this report), An experienced, $\mathrm{PhD}$ level qualitative researcher (either AA or CM) led each of the focus group discussions, and AA conducted the telephone interviews at KPNC. Either an ostomy nurse (JC) or urologic oncologist (SG) was present at the focus groups to address any clinical questions. All interviews were audio recorded and transcribed.

\section{Analyses}

The goal of the analysis was to describe a general framework for understanding diverse experiences. To accomplish this, we analyzed transcripts to identify commonalities and differences by: 1) urinary diversion type; and 2) health system. We used a modified grounded theory approach for analysis [22] to identify themes across the dataset, complemented by analytic memos and comparative analysis.

\section{Theme identification}

Starting with the KPNC dataset, the lead author reviewed all transcripts, and created a list of topics that were then consolidated into a set of unique themes and domains that were discussed with the research team. The lead author, a PhD-level anthropologist and qualitative researcher (CM), and a research associate (JD) then returned to the transcripts and the interviews and coded segments of KPNC focus groups associated with those themes and domains. For the Moffitt dataset, the lead author and another research associate (AF) slightly revised that list of themes and domains, and AF returned to the transcripts to code them accordingly. MG, a PhD-level nurse researcher, reviewed the Moffit 
1. Sometimes before bladder cancer surgery, surgeons talk to patients about the different ways that urine will leave their body after surgery. Did you and your surgeon or any other health care provider have that kind of discussion, about whether you'd have a neobladder or urostomy before you had your surgery? Who did you have that discussion with? What was it like?

2. Before your surgery, what was your understanding of the changes you would need to make in your daily life once you had a (neobladder/urostomy as appropriate)? What did you expect that having a (neobladder/urostomy as appropriate) would be like?

3. When you were recovering in the hospital from the surgery, what did you find most challenging? Who or what did you find most helpful?

4. Were there some of the things that you needed help with when you got home?

5. Now l'd like us to take some time to discuss your daily routine of having a (neobladder/urostomy as appropriate).

6. Do you have any extra costs because of your (neobladder/urostomy as appropriate), such as paying for adult diapers, ostomy pouches, catheters or needing to pay for additional drycleaning or laundry, that you didn't have before the surgery?

7. For some of you, having the (neobladder/urostomy as appropriate) may still be very challenging, and for others of you, having a (neobladder/urostomy as appropriate) may now be the "new normal." I'd like us to take some time now so that everyone can have a chance to talk about what it's like adjusting to a (neobladder/urostomy as appropriate).

8. For some people after their surgery, resuming physical intimacy and/or sexual activity becomes important. Can you tell me about how physical intimacy and sexual activity have been affected by your surgery and treatment?

9. Are there other areas of your life that your (neobladder/urostomy as appropriate) has affected?

10. If you had a close friend or family member who was going to have their bladder removed, what advice, based on your experiences, would you give him/her about learning to live with it? In other words, is there anything you know now that you wish you would have known when you first had your urinary diversion?

Fig. 1. Discussion prompts for focus groups and individual interviews. 
dataset and confirmed that the revised themes and domains were appropriate and correctly applied to the data.

\section{Analytic memos}

Analytic memos provided another approach to summarize key insights from each focus group, as well as the set of individual interviews. AA, a PhD-level sociologist who collected the KPNC data, wrote analytic memos about the KPNC data. $\mathrm{SG}$, a urologic oncologist experienced in qualitative methods and who was present at the Moffitt focus groups, wrote analytic memos about the Moffitt data. MG, who had independently read all the data, also reviewed and commented on the analytic memos.

\section{Comparative analysis}

To write this manuscript, which focused on crosssite comparisons, take-aways from analytic memos (which were written by site) and summaries of the coded data from each site were reviewed by the lead author (CM) and a research associate (NB).

\section{RESULTS}

\section{Participants}

On average, participants at the integrated system were 70 years old (range: $47-87$ years). Their surgeries took place an average of 2.6 years before their study participation (range: 1-6 years). Each focus group included two racial/ethnic minority participants; four of the women we interviewed by telephone were non-Hispanic white and one was of unknown racial/ethnic background.

The average age of the comprehensive cancer center patient participants was 68 years (range: $38-93$ years). Their surgeries took place an average of 2.1 years before study participation (range: $0-5$ years). One patient participant self-identified as a racial or ethnic minority, all others identified as non-Hispanic white.

\section{Domains of patient experience}

We identified 12 themes from the interviews that we grouped into four domains of post-operative experience: (1) decision-making about and preparation for urinary diversion; (2) surgery and post-operative recovery; (3) mastery of self-care for the urinary diversion; and (4) reintegration of identity and participation in major life activities (Fig. 2). These domains correspond to major phases in the clinical course for patients undergoing cystectomy with urinary diversion and also conformed to how patients described their experiences. Specific quotes related to each theme are found in Table 1.

\section{Decision-making}

Across both health systems, participants described highly variable experiences in decision-making about their cystectomy and urinary diversion. Some felt overwhelmed by the amount of information they were given, while others felt that their doctors did not inform them adequately about their options. Some recalled that their urologist expressed a strong preference for one type of diversion; others were presented with more neutral information. Perceptions about the benefits and disadvantages of different diversion types were also not consistent. For example, ileal conduits and neobladder diversions were each described as simpler to live with by different urologists.

Participants also varied in whether they preferred to follow a clear recommendation from their provider or whether they wanted a more active role in the decision. Some patients sought second opinions from other urologists, sought additional information online, or reported consulting with others. While patients at both health systems reported some similar experiences, integrated system patients described their diagnosis and decision-making processes as relatively straightforward. By contrast, patients who were treated at the cancer center often had initial unsatisfying experiences in other health systems before seeking a second opinion at the cancer center; others saw different providers within the cancer center for treatment before their diversion surgery. Some described poor communication or follow-up between these previous providers.

\section{Surgery and post-operative recovery}

With few exceptions, the immediate postoperative recovery period was a challenging time for patients. Patients and caregivers struggled to learn self-care skills at the hospital that would prepare them for a smooth transition to home. Patients were often unaware of potential issues they might have with bowel function or other complications that can arise after surgery-infection, hernia, and urinary tract 


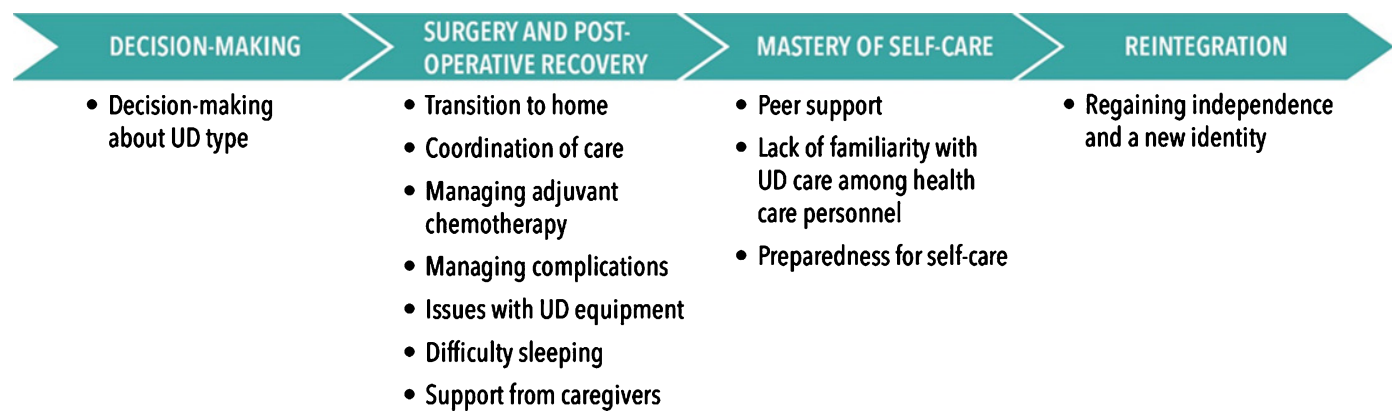

Fig. 2. Domains and themes of patient experience.

infection (UTI). Patients in both systems reported wanting more information and supplies when being discharged from the hospital. However, some participants described being overwhelmed by supply samples and unsure how to choose and order the right supplies for themselves.

A primary challenge for many patients was learning to use self-care equipment during the recovery period both in the hospital and once they had returned home. Some patients at the integrated system reported having issues with nurses and other staff who were unfamiliar with their diversion or the related equipment or felt that they were expected to learn self-care after just a few demonstrations. Patients at both systems reported that they were often unsure if they were doing self-care correctly, especially after returning home, and weren't sure what problems required medical attention and what was a normal part of their body adapting to the change. In addition, patients struggled to learn how to sleep with their new equipment while dealing with nighttime leakage.

One area where patient experience differed notably between systems was coordination of care. Patients in the integrated system reported a variety of experiences with care coordination after surgery. While some felt they received excellent care, others faced challenges: in some cases, no stoma nurse was available in a patient's city; in other cases, providers treating surgical complications appeared unfamiliar with urinary diversions. In general, patients in the integrated system did not always know who to contact to get their needs met after leaving the hospital. Patients at the cancer center did not mention as many care coordination or follow-up care challenges and tended to have a clear understanding about who they should reach out to with problems and concerns.
After returning home, some patients began adjuvant chemotherapy shortly after the surgery, further complicating their recovery. Participants reported a wide variety of information needs that they wished had been met before surgery: many did not feel that they had been given adequate information about what to expect from chemotherapy, recovery, and transitioning to home, or that they weren't given enough information about self-care. These struggles were common for patients in both health care systems.

Family caregivers often took charge of changing ostomy equipment, managing medication regimens, and providing other support after patients returned home. These caregivers frequently learned by observing nurses in the hospital, but they were not typically given any formal training or resources during the postoperative period.

\section{Mastery of self-care}

After the initial recovery period, patients gradually became experts who were comfortable and skilled with self-care. The pathway to becoming an expert was highly variable but was a uniform goal.

Some patients had home care nurse visits a for a month or two after surgery, and gradually assumed more and more of their self-care; many found these visits very helpful. Participants sought out additional information related to their urinary diversion through Internet searches, blogs, and YouTube videos. Some of the most valuable help came from conversations with others who had gone through a similar surgery, whether those people were friends and relatives, support group members, or contacts given by a provider. Participants commented that even the focus group discussions yielded additional information and tips on self-care. 
Table 1

Selected patient quotes illustrating phases of urinary diversion experience

DECISION-MAKING

SURGERY \& POST-OPERATIVE RECOVERY

Transition to home

Coordination of care

Managing adjuvant chemotherapy

Managing complications

Issues with UD equipment

Difficulty sleeping
"I had the situation where he explained - like I said, he explained everything to me. He explained the positives and negatives of either way. And I selected not (to have) the neo-bladder. Because one of the things that I was concerned about was the leakage." (integrated health system)

"But I just didn't feel as I had enough information. I changed my mind within 8 hours of the operation." (cancer center)

"We had really awesome home health care and people came for us every day and they changed out the wafers periodically so I got to kind of learn from somebody who actually knew what to do what they do how to avoid problems and I think we've all had a few screw ups over the last couple years and for whatever reason never figured it out but basically it's been good." (cancer center)

Patient 1: "Well, we had home care for a week or two."

Patient 2: "Well, I didn't have that ... they said, if I'm not able to get to the hospital, then they would do that, but I had to send an affidavit that I was, you know, bedridden and couldn't get there."

Patient 1: I had three, four weeks of it.

Patient 3: I did too.

Patient 4: They asked me and I said no. (integrated health system)

"I went to BCG and all the other multiple cystos up to that point, but one day I was in there for a cysto and a doctor came in and said, "oh I guess we'll put the stoma right here." And I said, "What?" It was like out of the blue, and then he said, "... we're going to take your bladder." It was like a total shock." (cancer center patient talking about experiences before coming to the cancer center)

"Inside the urology community, they know about neobladders, but the minute you get outside, they haven't a clue." (integrated health system)

"I wish that my urologist would have talked to my oncologist more. Because when I found out I had bladder cancer, they just said, "you know, you got to have the R.C., blah blah blah blah." And then ... after the surgery, I went to see my Oncologist ... and he said that, if he would have known, if they were talking to each other, I would have had some chemo before the surgery, then I wouldn't have had to have as much chemo at the back end." (integrated health system)

"The thing that kind of threw me a little bit off was the chemo part of it seem[ed] to be harder than the surgery part of it... I was just thinking I'll be back at work in two weeks and it didn't work out that way obviously it was probably closer to 2 months before I got back to ... working." (cancer center)

"I'd like to have had more information on edema ... and hernias. I had a hernia that started about 2 months after surgery and I'd deal with it with a hernia belt and my leg started swelling maybe 3 months after. I didn't know that was going to happen." (cancer center)

"I had a lot of issues after surgery because I developed a fistula after surgery, and they did not want to go in and do surgery again. So I was on TPN for two months... it's a way to feed yourself through an IV because some people think fistulas heal better if you don't put any food in your mouth." (integrated health system)

"I wound up with like a rash around my stoma... And it was weeping, and so pouches would fall off ... so each time I'd get in the shower and wash everything off, try patting all dry and everything and try it again... I'd put on a pouch. It would fall off because of the weeping. I'd be a mess, and a lot of it was about 3:00 in the morning. I had one pouch left." (integrated health system)

"The nurse at the hospital would show me but what I should have had was like a mirror to see what she was doing. I couldn't see over her fingers." (cancer center)

I did the night bag ... a and it was just so restrictive ... you can't really move around. So you're not really sleeping well, and then a couple times [the night bag] dislodged. So then I found I had to tape it to stay on, and so finally I said I'm just not going to do this. I'm just going to use my kitchen timer, and I'm going to set it for two hours. I'm going to get two hours sleep, and then I'll just wake up when it goes off and I'll go to the bathroom. (integrated health system) "I found it helpful when I got home I slept in a recliner for like 2 months [others agree]. It's hard for me to sleep anywhere else." (cancer center) 
Table 1

(Continued)

\begin{tabular}{|c|c|}
\hline Support from caregivers & $\begin{array}{l}\text { "My wife said, "No." "I love you, but I'm not doing that. [Flushing tubes and changing } \\
\text { bandages]." (integrated health system) } \\
\text { "I have never changed my bag since I came out of the hospital. She [wife] does it all the time. } \\
\text { I know it may be a problem for her but it's a godsend for me." (cancer center) }\end{array}$ \\
\hline \multicolumn{2}{|l|}{ MASTERY OF SELF-CARE } \\
\hline Peer support & $\begin{array}{l}\text { "I go do BCAN every day (Bladder Cancer Advocacy Network). You can talk to others. I saw } \\
\text { a YouTube video of this man changing his bag, and what he did... He said your stoma should } \\
\text { be dry before you put on your bag." (integrated health system) } \\
\text { "I went to one (support group). I hated it. I enjoyed talking to people and learning about their } \\
\text { situation, but at the end of it, there was the word of the day, like compassion - people sort of } \\
\text { speak on the word and then we held hands." (cancer center) }\end{array}$ \\
\hline $\begin{array}{l}\text { Lack of familiarity with UD care among } \\
\text { health care personnel }\end{array}$ & $\begin{array}{l}\text { "The nurses, even the stoma nurses, really don't know about day to day. We had to work our } \\
\text { way through it. I (used a movie) on YouTube." (integrated health system) } \\
\text { "I mean, I was in there and I didn't have anybody to come over to check this bag and when I } \\
\text { had a vac and that's supposed to be changed and they never ... they never, they were } \\
\text { supposed to send somebody up here to change it and they never changed, I just sat there for a } \\
\text { week with that thing leaking all over." (cancer center) }\end{array}$ \\
\hline Preparedness for self-care & $\begin{array}{l}\text { "When it comes to the neobladder in particular, they did a good job in the hospital or showing } \\
\text { me how to use ... the syringe and the catheters and all that stuff, but when you get home } \\
\text { (laughs) ... And now you've got all this stuff you're trying to figure out how am I going to do } \\
\text { this and that whatever, I think videos probably would have been very helpful." (cancer center) } \\
\text { "Nice people. Knew what they were talking about. Of course, it's still a learning curve. I } \\
\text { mean, they never told you that whenever you put stuff on, take your hair dryer out and make } \\
\text { sure everything's dry..." (integrated health system) }\end{array}$ \\
\hline REINTEGRATION & $\begin{array}{l}\text { "If you're tough, you can manage it. If you're not, I don't know... I would say it's not as bad as } \\
\text { what you think. Because the ordeal that I went through was really a lifetime challenge for me. } \\
\text { I had no experience, no real expectations, and everything hit me right in the moment. } \\
\text { Recovery was difficult. Changing my lifestyle, my clothing. It is hard. Having gone through } \\
\text { that gave me some different expectations... I can perform at a much higher level that I thought } \\
\text { I could." (integrated health system) } \\
\text { "We spent the first year of healing and regaining in general and then this past year we started } \\
\text { trying to alter the life as needed and um several aspects that we're still struggling with in our } \\
\text { daily life." (cancer center) }\end{array}$ \\
\hline
\end{tabular}

Patients at both health care systems shared experiences in which hospital staff or home health care nurses did not know enough about a patient's urinary diversion to help manage their ostomy or catheterization equipment.

Participants talked about making choices about the type of equipment that worked best, becoming comfortable with catheterization, and learning to treat and prevent skin problems. Several patients noted that there was an inevitable "learning curve" to becoming an expert at self-care regardless of the quality of support one receives from their providers, in part because every patient faces different challenges.

\section{Reintegration}

Participants talked about the challenges involved with the changes to their body and daily routine, as well as difficulties coping with their cancer diagnosis. For some, life after cystectomy entailed enduring the loss of valued physical activities, employment, and independence; others found that they didn't have to make major changes to their lifestyle. Some expressed difficulties with traveling, a lack of spontaneity in everyday activities since immediate access to toilet facilities may be essential, loss of stamina and endurance, sexual inability, and lost friends. Participants stated that adjusting to these challenges was part of regaining self-confidence and self-acceptance. Several also noted positive changes in their outlook and personality that they attributed to the experience of surviving a major illness.

\section{DISCUSSION}

Through focus groups and interviews with total cystectomy patients in two very different types of health care systems, we identified substantial variability within and across settings in the experiences and challenges patients faced. However, 
we found that participants consistently described a transformative process with four domains of experience: pre-operative decision-making, surgery and post-operative recovery, mastery of self-care, and eventual reintegration into the activities of daily life. Across a wide range of experiences, these domains reflect a consistent pathway that was salient to the men and women we interviewed regardless of health system or urinary diversion type (see Fig. 2). These four domains and the themes within each domain provide insight into critical patient experiences and outcomes beyond straightforward measures of functioning and quality of life. These include decision making and decision appraisal, satisfaction with care, ostomy or neobladder-related self-care, informal caregiving, self-efficacy, and personal meaning in illness experience. These aspects of patient experience are often overlooked in comparative studies about different urinary diversions, which have focused more on health-related quality of life measures and clinical outcomes $[23,24]$.

While patients in both health systems reported similar treatment and recovery journeys and highlighted the same needs for information and support throughout the process, a few key differences emerged between the two health systems. First, the diagnosis and decision-making journey tended to be more consistent for patients in the integrated system (KPNC) than for cancer center (Moffitt) patients. Second, patients in the two systems reported different experiences of follow-up care: cancer center patients had a more straightforward process for getting post-operative problems addressed and were less likely to experience challenges with hospital staff care coordination or treatment for complications. These differences likely reflect realities of receiving care at a cancer center and an integrated health system. Because many patients turn to cancer centers for a second opinion or through referral, they are not typically being treated by their primary urologist, and represent a somewhat different population of individuals than those treated in an integrated system, where patients are often treated by the same urologist throughout the course of their bladder cancer. In an integrated system, the number of patients receiving cystectomies is much lower than in a comprehensive cancer center, and there are many possible entry points to care after surgery; depending on the need, patients might contact or be routed to a general advice nurse, a primary care provider, or a provider in the urology department. By contrast, patients at the cancer center rely on a designated urologic oncology team for all follow-up care. These differences nonetheless highlight the importance of care coordination to patients in both systems throughout the treatment journey. More research is needed to examine how health systems can encourage patient-centered care for treatment decisions, perioperative and post-operative care, and longer-term survivorship.

Because many patients facing cystectomy are candidates for more than one type of urinary diversion, more research on decision making about diversion types is needed. A recent study of cystectomy patients who received care at a major academic medical center found that these patients' highest priorities in treatment decision-making were receiving treatment from experts and making decisions based on personal preferences [16]. These priorities differed sharply from those expressed by integrated system patients in our study, who infrequently sought second opinions or additional consultations to help decide between cystectomy options. This contrast illustrates the importance of collecting diverse patient perspectives on cystectomy and urinary diversion.

This study had the strength of comparing patient outcomes across two very different health systems while demonstrating numerous common themes in the treatment and recovery experiences of patients, suggesting wide generalizability of our findings. However, we did not directly assess patients treated in other settings (such as fee-for-service private practice), and more research is necessary to determine how our findings generalize to these patients. Another potential limitation of our study is that participants were as much as 6 years removed from their surgery and may have experienced some recall bias. These limitations should be addressed by future research.

Our study, which elicited patient perspectives on the process of urinary diversion surgery and recovery across two health care settings, highlighted several care gaps. In decision-making, for example, across both health systems, participants described highly variable experiences. Before making this major decision, some patients said they received too little information; some said they received too much. Some patients were told an ileal conduit was "simpler to live with"; others were told the opposite - that a neobladder would be simpler. In the recovery phase, many patients found learning to use self-care equipment a challenge. Patients at both health systems felt unprepared for the transition to home and often weren't sure what problems required medical attention and what 
was normal. Our study underscores that cystectomy patients often do not receive adequate information, preparation, or training in how to care for themselves post-surgery $[14,15,25]$.

This study also contributes a grounded theoretical framework for describing the treatment and recovery experience of patients undergoing cystectomy and urinary diversion (see Fig. 2). Our results highlight that while patient experiences are variable, they can be understood as part of a process encompassing decision-making, recovery, developing self-care expertise, and reintegration. This general framework suggests patient-centered domains for research and care improvement efforts that transcend urinary diversion type, gender and health system contexts.

\section{ACKNOWLEDGMENTS}

We would like to thank David Aaronson, Assistant Chief of the Department of Urology at Kaiser Permanente East Bay, for his support in conducting this research.

\section{CONFLICT OF INTEREST}

The authors have no conflicts of interest to report.

\section{FUNDING}

This project was funded by the National Institutes of Health, Grant Number: 1R01CA164128.

\section{REFERENCES}

[1] Stein JP, Lieskovsky G, Cote R, Groshen S, Feng AC, Boyd $\mathrm{S}$, et al. Radical cystectomy in the treatment of invasive bladder cancer: Long-term results in 1,054 patients. J Clin Oncol. 2001;19(3):666-75.

[2] Gerharz EW, Mansson A, Hunt S, Skinner EC, Mansson W. Quality of life after cystectomy and urinary diversion: An evidence based analysis. J Urol. 2005;174(5):1729-36.

[3] Porter MP, Penson DF. Health related quality of life after radical cystectomy and urinary diversion for bladder cancer: A systematic review and critical analysis of the literature. $\mathrm{J}$ Urol. 2005;173(4):1318-22.

[4] Porter MP, Wei JT, Penson DF. Quality of life issues in bladder cancer patients following cystectomy and urinary diversion. UrolClin North Am. 2005;32(2):207-16.

[5] Nabi G, Yong SM, Ong E, McPherson G, Grant A, N'Dow J. Is orthotopic bladder replacement the new gold standard? Evidence from a systematic review. J Urol. 2005;174(1): 21-8.
[6] Hardt J, Filipas D, Hohenfellner R, Egle UT. Quality of life in patients with bladder carcinoma after cystectomy: First results of a prospective study. Qual Life Res. 2000;9(1): $1-12$.

[7] Henningsohn L, Wijkstrom H, Dickman PW, Bergmark K, Steineck G. Distressful symptoms after radical cystectomy with urinary diversion for urinary bladder cancer: A Swedish population-based study. Eur Urol. 2001;40(2): 151-62.

[8] Hautmann RE, Volkmer BG, Schumacher MC, Gschwend JE, Studer UE. Long-term results of standard procedures in urology: The ileal neobladder. World J Urol. 2006;24(3):305-14.

[9] Botteman MF, Pashos CL, Hauser RS, Laskin BL, Redaelli A. Quality of life aspects of bladder cancer: A review of the literature. Qual Life Res. 2003;12(6):675-88.

[10] Dutta SC, Chang SS, Coffey CS, Smith JAJ, Jack G, Cookson MS. Health related quality of life assessment after radical cystectomy: Comparison of ileal conduit with continent orthotopic neobladder. Journal of Urology. 2002;168:164.

[11] Hobisch A, Tosun K, Kinzl J, Kemmler G, Bartsch G, Holtl $\mathrm{L}$, et al. Life after cystectomy and orthotopic neobladder versus ileal conduit urinary diversion. SeminUrolOncol. 2001;19(1):18-23.

[12] Smith AB, Chisolm S, Deal A, Spangler A, Quale DZ, Bangs $\mathrm{R}$, et al. Patient-centered prioritization of bladder cancer research. Cancer. 2018. doi: 10.1002/cncr.31530

[13] Strauss A, Corbin JM. Grounded Theory in Practice. Thousand Oaks, CA: SAGE Publications; 1997.

[14] Mohamed NE, Chaoprang Herrera P, Hudson S, Revenson TA, Lee CT, Quale DZ, et al. Muscle invasive bladder cancer: Examining survivor burden and unmet needs. J Urol. 2014;191(1):48-53. doi: 10.1016/j.juro.2013. 07.062

[15] Mohamed NE, Pisipati S, Lee CT, Goltz HH, Latini DM, Gilbert FS et al. Unmet informational and supportive care needs of patients following cystectomy for bladder cancer based on age, sex, and treatment choices. Urol Oncol. 2016;34(12):531.e7-.e14. doi: 10.1016/j.urolonc. 2016.06.010

[16] Berry DL, Nayak M, Halpenny B, Harrington S, Loughlin KR, Chang P, et al. Treatment Decision Making in Patients with Bladder Cancer. Bladder Cancer. 2015;1(2):151-8. doi: $10.3233 /$ blc- 150029

[17] Foley G, Timonen V. Using grounded theory method to capture and analyze health care experiences. Health Serv Res. 2015;50(4):1195-210. doi: 10.1111/1475-6773.12275

[18] Strauss A, Corbin J. Basics of Qualitative Research: Grounded Theory Procedures and Techniques. Newbury Park, CA: Sage Publications; 1990.

[19] Luborsky MR, Rubinstein RL. Sampling in qualitative research: Rationale, issues, and methods. Res Aging. 1995;17(1):89-113. doi: 10.1177/0164027595171005

[20] Patton M. Qualitative Evaluation and Research Methods. Thousand Oaks, CA: SAGE Publications; 2002.

[21] Grant M, McMullen CK, Altschuler A, Mohler MJ, Hornbrook MC, Herrinton LJ, et al. Gender differences in quality of life among long-term colorectal cancer survivors with ostomies. Oncol Nurs Forum. 2011;38(5):587-96. doi: 10.1188/11.onf.587-596

[22] Schreier M. Qualitative content analysis. In U. Flick ed. London: Sage; 2014, pp. 170-83.

[23] Ghosh A, Somani BK. Recent trends in postcystectomy health-related quality of life (QoL) favors neobladder 
diversion: Systematic review of the literature. Urology. 2016;93:22-6. doi: 10.1016/j.urology.2015.12.079

[24] Tyson MD 2nd, Barocas DA. Quality of life after radical cystectomy. Urol Clin North Am. 2018;45(2):249-56. doi: 10.1016/j.ucl.2017.12.008
[25] McMullen CK, Hornbrook MC, Herrinton LJ, Altschuler A, Grant M, Wendel C, et al., editors. Kaiser Permanente Northern California improving survivorship care for longterm colorectal cancer survivors: Key findings of a 5-years study, 2009. 